\title{
One-pot preparation of three-component oil-in-water high internal phase emulsions stabilized by palm-based laureth surfactants and their moisturizing properties.
}

\begin{abstract}
In the present study, olive and olein oils had been used for the preparation of threecomponent high internal phase emulsions with oil volume fraction of more than 0.77 stabilized by palm-based laureth surfactants for the first time, respectively. These emulsions were easily prepared by one-pot homogenization. The critical micelle concentration and Gibbs energy of the as-synthesized surfactants were determined and discussed. Likewise, the morphology, structural properties, stability and hydration efficacy of the as-prepared emulsions were investigated. Droplet size distribution observed from the optical micrographs was in agreement with the light scattering results which suggested that droplet size increased with increasing ethylene oxide chain length. The rheological measurements of the emulsions at room $\left(25^{\circ} \mathrm{C}\right)$ and elevated $\left(40^{\circ} \mathrm{C}\right)$ temperatures were interpreted to give clear and direct explanation on the structure and stability of the emulsions. The hydration efficacy of the emulsions was examined in vivo using a corneometer. Both the emulsions containing olive and olein oils, respectively exhibited high stability as indicated by the rheological measurements and the structural properties did not differ from one another. However, olein oil's hydration efficacy was higher than olive oil's, suggesting that olein oil could well be a potential moisturizing lipid which might interest the dermatologists.
\end{abstract}

Keyword: Internal phase emulsion; Surface active agents; Colloids. 